\section{REGIONAL DELIVERY OF A TLR9 AGONIST TO BOOST CHECKPOINT INHIBITOR RESPONSIVENESS IN LIVER METASTASES}

${ }^{1}$ Chandra Ghosh*, 'Kyle O'Connell, 'Kara Heatherton, 'Jason Laporte, 'Prajna Guha, ${ }^{2}$ Bryan Cox, ${ }^{1}$ Steven Katz, ${ }^{2}$ Steven Katz. ${ }^{1}$ Roger WIlliams Medical Center, Providence, RI, USA; ${ }^{2}$ Trisalus Life Sciences, Westminster, CO, USA

Background Class C TLR9 agonists, CpG oligodeoxynucleotides (ODNs) enhance responsiveness to anti-PD1 therapy in solid tumors through favorable modulation of the tumor microenvironment (TME) [1]. Recently, we reported that regional delivery of a TLR9 agonist eliminated myeloid derived suppressor cells (MDSC) and promoted pro-inflammatory/anti-tumorigenic M1 macrophage programming in the TME of liver metastases (LM) [2]. Further, we found enhanced TLR9 activation in LM following regional TLR9 agonist infusion compared to the systemic treatment. We hypothesize that regional delivery of a TLR9A into LM will enhance the responsiveness to systemically infused anti-PD1 therapy.

Methods In this study, we treated mice with established MC38-CEA-Luc LM with ODN-2395 (30 $\mathrm{g} / \mathrm{mouse})$ regionally with or without anti PD-1 antibody $(250 \mu \mathrm{g} / \mathrm{mouse})$ intraperitoneally.

Results Control of LM growth (Figure 1) was significantly higher with combinatorial treatment as compared to anti-PD1 $(\mathrm{p}<0.01)$ or PBS treatments $(\mathrm{p}<0.05)$. To study the impact of TLR9 activation on human MDSC, we treated healthy donor PBMCs with ODN-2395 or SD101. We found that both reduced the hu-MDSC (CD11b+CD33+HLADR-) population in a dose-dependent manner with an increase in PD-L1 expression as determined by flow cytometry (FC) analysis (Figure 2). Moreover, by using Luminex, demonstrated that ODN-2395 and SD101 enhanced expression of IL 29, IFN $\alpha$, and NFKB, along with downstream cytokines IL 6 and IL 10. To investigate the effect of SD101 in modulating the differentiation of huMDSC from huPBMC, we treated huPBMC with IL6+GM-CSF in the presence or absence of SD101. By FC analysis, we found that SD-101 blocked huMDSC development induced by IL6+GM-CSF, preferentially limited the more immunosuppressive monocytic MDSC subtype, and drove M1 macrophage polarization. Treatment of SD101 only once for $48 \mathrm{hrs}$ was sufficient to inhibit huMDSC differentiation for two weeks.

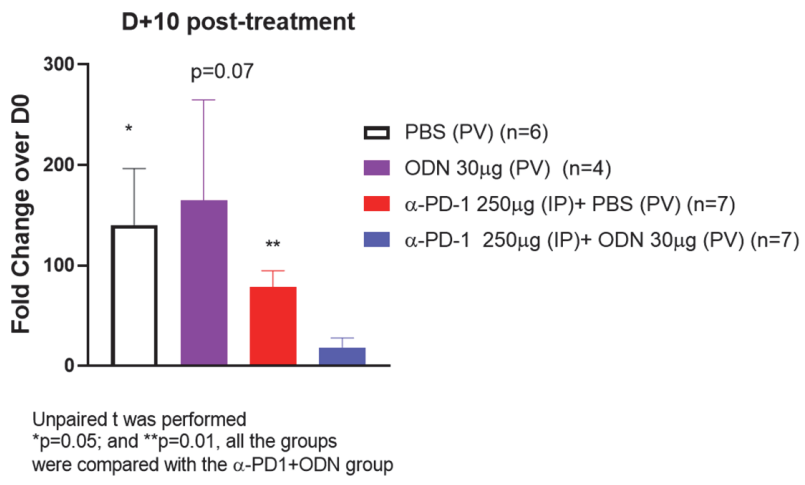

Abstract 607 Figure 1 Combinatorial treatment of CPI and ODN's reduces $t$

$\mathrm{PV}=$ portal vein; IP = intraperitoneal.

Eight to twelve weeks old male C57/BL6 mice were challenged intra-splenic with 0.5e6 MC38-CEA-Luc cells for a week. Bioluminescence value was determined by IVIS on D0, and mice were randomized accordingly and treated with 30 $\mu \mathrm{g} /$ mouse ODN2395 via PV with or without $250 \mu \mathrm{g} / \mathrm{mouse}$ anti-PD1 antibody via IP on D0, D+3 and D+6. PBS served as the vehicle (Veh) control and administered via PV. Fold change of the tumor burden was calculated based on D0 baseline bioluminescence. Tumor progression was analyzed unpaired t test among groups. ("p <0.05).

\section{A. Hu MDSC}



\section{B. MFI PD-L1}

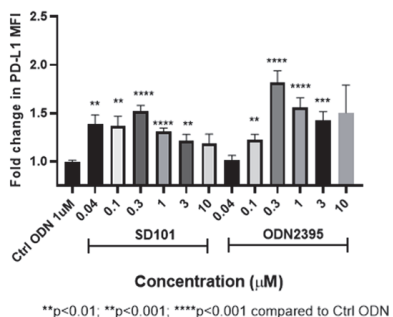

Abstract 607 Figure 2 Human PBMC treated with ODN2395 and SD101 reduces

Ctrl = control; MDSC = ODN = oligodeoxynucleotide' PBMC = peripheral blood monocytes.

Human PBMC were isolated from the Leukoreduction system chamber. 1e6/ml PBMCs were treated with increasing concentrations $(0.04-10 \mu \mathrm{M})$ SD101, ODN2395 along with ctrl ODN5328 $(1 \mu \mathrm{M})$ for 48 hours. Panels A and B: MDSC population and their corresponding PD-L1 expression were evaluated $(n=12)$. Four donors with three replicates were used. Data represented as mean \pm SEM.

Conclusions Both the in vitro and in vivo findings suggest that regional TLR9 stimulation in a model of LM improves responsiveness to systemic anti-PD-1 therapy through elimination of MDSC, and the effect on huMDSC was confirmed in vitro. Increased PDL-1 expression in response to TLR9 stimulation among MDSC may further enhance the anti-PD-1 effect. Therefore, combing regional infusions of a TLR9 agonist with systemic anti-PD-1 agents may be a promising approach for liver tumor treatment.

\section{REFERENCES}

1. Wang, S., et al., Intratumoral injection of a $\mathrm{CpG}$ oligonucleotide reverts resistance to PD-1 blockade by expanding multifunctional CD8+ T cells. Proc Natl Acad Sci U S A, 2016. 113(46): p. E7240-E7249.

2. Ghosh CC, H.K., O'Connell K, Laporte J, Guha P, Cox B, Jaroch D, Katz SC, Regional administration of class C CpG Oligodeoxynucleotides results in superior intrahepatic TLR9 activation and immunomodulation compared to systemic infusion, Abstract: AACR Annual Meeting. 2021

http://dx.doi.org/10.1136/jitc-2021-SITC2021.607 